\title{
Perfil biopsicossocial de crianças e adolescentes institucionalizados
}

\author{
Biopsychosocial profile of institutionalized children and teenagers \\ Perfil biopsicosocial de niños y adolescentes institucionalizados
}

Camila Cristina Costa ${ }^{1 *}$, Elaine Cristina Dias Franco ${ }^{1}$, Thaissa Magela dos Santos ${ }^{1}$, Edilene Aparecida Araújo da Silveira ${ }^{1}$, Marcela Silva Carvalho¹, Maria Alice Aparecida Resende¹.

\section{RESUMO}

Objetivo: Caracterizar, quanto aos aspectos biopsicossociais, a população de crianças e adolescentes em situação de acolhimento institucional em um município da região centro-oeste de Minas Gerais. Métodos: Estudo do tipo exploratório descritivo. Os participantes foram 11 crianças e 12 adolescentes institucionalizados em Abrigos de um município de Minas Gerais. A coleta de dados ocorreu de outubro a dezembro de 2018, por meio de um roteiro semiestruturado inspirado no questionário da Pesquisa Nacional de Saúde do Escolar - PeNse. Resultados: Quanto ao consumo de alimento em cinco dias ou mais na semana, todos os participantes, de ambos os sexos, consomem diariamente feijão e frutas; $81 \%$ do sexo masculino comem guloseimas e $50 \%$ do sexo feminino tomam refrigerante; $53 \%$ dos participantes praticam atividade física e 10\% assistem televisão mais de duas horas por dia. Em relação a tabacos e drogas, $53 \%$ disseram já ter experimentado alguma bebida alcóolica e 15\% usaram drogas ilícitas uma vez na vida. Conclusão: O contexto familiar composto de situações de risco e vulnerabilidades permite que as crianças e adolescentes tenham suas histórias de vida permeadas por condições socioeconômicas precárias, bem como aproximações diretas e indiretas com consumo de drogas lícitas e ilícitas.

Palavras-chave: Adolescente, Criança, Institucionalização, Atenção à Saúde.

\begin{abstract}
Objective: To characterize, as regards biopsychosocial aspects, the population of children and adolescents in institutional care in a municipality in the Midwest region of Minas Gerais. Methods: Descriptive exploratory study. The participants were 11 children and 12 adolescents institutionalized in shelters in a municipality of Minas Gerais. Data collection took place from October to December 2018, through a semi-structured script inspired by the questionnaire of the National School Health Survey - PeNse. Results: As for food consumption on five days or more a week, all participants, both sexes, consume beans and fruits daily; $81 \%$ males eat goodies and $50 \%$ females drink soda; $53 \%$ of participants practice physical activity and $10 \%$ watch television more than two hours a day. Regarding tobacco and drugs, 53\% said they had tried some alcohol and $15 \%$ used illicit drugs once in their lifetime. Conclusion: The family context composed of risk and vulnerability situations allows children and adolescents to have their life histories permeated by precarious socioeconomic conditions, as well as direct and indirect approaches with licit and illicit drug use.
\end{abstract}

Key words: Adolescent, Child, Institutionalization, Health Care.

1Universidade Federal de São João del Rei (UFSJ), Divinópolis-MG. *E-mail: camilacosta542@gmail.com Projeto de Iniciação Científica aprovado no Edital 009/2017/PROPE - PIBIC/FAPEMIG/UFSJ SUBMETIDO EM: 9/2019 | ACEITO EM: 9/2019 | PUBLICADO EM: 10/2019 


\section{RESUMEN}

Objetivo: caracterizar, en lo que respecta a aspectos biopsicosociales, la población de niños y adolescentes en atención institucional en un municipio de la región del Medio Oeste de Minas Gerais. Métodos: estudio exploratorio descriptivo. Los participantes fueron 11 niños y 12 adolescentes institucionalizados en albergues en un municipio de Minas Gerais. La recopilación de datos tuvo lugar de octubre a diciembre de 2018, a través de un guión semiestructurado inspirado en el cuestionario de la Encuesta Nacional de Salud Escolar - PeNse. Resultados: En cuanto al consumo de alimentos durante cinco días o más a la semana, todos los participantes, ambos sexos, consumen frijoles y frutas diariamente; $81 \%$ de los machos comen golosinas y $50 \%$ de las hembras beben refrescos; El $53 \%$ de los participantes practica actividad física y el $10 \%$ mira televisión más de dos horas al día. Con respecto al tabaco y las drogas, el 53\% dijo que había probado el alcohol y el $15 \%$ usó drogas ilícitas una vez en su vida. Conclusión: El contexto familiar compuesto por situaciones de riesgo y vulnerabilidad permite a los niños y adolescentes tener sus historias de vida impregnadas por condiciones socioeconómicas precarias, así como enfoques directos e indirectos con el uso de drogas lícitas e ilícitas.

Palabras clave: Adolescente, Niño, Institucionalización, Atención a la Salud.

\section{INTRODUÇÃO}

As crianças e de modo especial, os adolescentes constituem um grupo prioritário para a promoção da saúde devido a comportamentos e vivências que são expostos e que são relacionados ao contexto histórico, social e cultural em que se dá o desenvolvimento desses grupos e que em graus variados os expõem a inúmeras situações de risco para a saúde (ZAPPE JG e DELL'AGLIO DD, 2016; MALTA DC, et al., 2010).

A adolescência configura-se como uma fase do ciclo vital que é permeada por importantes transformações biopsicossociais. No campo psicossocial denotam-se alterações nas relações escolares, a experimentação intensa de diversos sentimentos, a busca pela autonomia e independência familiar, bem como a predileção em experimentar novos comportamentos e vivências. Já no campo biológico são observadas, de forma evidente, alterações físicas e hormonais, dentre outras (MALTA DC, et al., 2010; MALTA DC, et al., 2014). Diante dessas transformações, estudos desenvolvidos nas últimas décadas revelam que o uso de álcool, cigarro e outras drogas; a adoção de comportamento sexual de risco, antissocial e suicida; hábitos alimentares não saudáveis e; prática inadequada de atividades físicas; dentre outros, representam importantes fatores de risco para a saúde dos adolescentes, bem como para o crescimento e desenvolvimento biopsicossocial destes (ZAPPE JG e DELL'AGLIO DD, 2016; MALTA DC, et al., 2014; MALTA DC, et al., 2010).

Neste contexto, desde a década de 1990 com apoio da Organização Mundial da Saúde (OMS) diversos países tem desenvolvido sistemas de vigilância baseado em inquéritos relacionados à saúde de escolares, nos quais são levantadas informações relativas à saúde física, emocional e psicológica destes, bem como a influência da escola, família e pais em seus cotidianos (MALTA DC, et al., 2014). No Brasil, algumas iniciativas de vigilância à saúde de adolescentes vem sendo realizadas a exemplo dos inquéritos relacionados ao uso de drogas psicotrópicos; ao Tabagismo em Escolares denominado de Vigescola e; a Pesquisa Nacional de Saúde do Escolar (PeNSE) que teve sua primeira edição em 2009 e desde então vem sendo realizada a cada três anos (MALTA DC, et al., 2010).

Em algumas situações esses comportamentos e vivências que envolvem a infância e a adolescência, considerados de risco, são potencializados devido aos contextos socioeconômicos, culturais e familiares fragilizados. Nesse caso entra em cena o Estatuto da Criança e do Adolescente (ECA) que ao delinear as Políticas Públicas de Proteção à Criança e Adolescente define dentre as estratégias de proteção, o acolhimento institucional previsto no Estatuto da Criança e do Adolescente como uma medida protetiva excepcional e provisória (BRASIL, 1990). A institucionalização deve se tornar uma opção quando a família não exerce sua função de protetora e apoiadora do menor, sendo que atualmente, de acordo com o Cadastro 
Nacional de Crianças Acolhidas, estão em casas de acolhimento 43.475 internos com idades que variam de zero a 19 anos (DINIZ IA, et al., 2018, BRASIL, 2019).

Estudos tem demonstrado que as crianças e adolescentes acolhidos tem em seus contextos familiares e de vivências a presença importante de fatores de risco à saúde como tabagismo, uso de álcool e drogas, violência, comportamento sexual de risco, comprometimento social e afetivo, dentre outros, que são considerados na determinação do acolhimento como medida protetiva. (DIAS MSL e SILVA RSB, 2012 ; FERREIRA VV, et al, 2014; FUKUDA CC, et al., 2013).

Entende-se que conhecer como vivem e se comportam as crianças e adolescentes institucionalizados, sob diversos aspectos, possibilita a identificação da magnitude e da distribuição de importantes fatores de risco à saúde, ancorando informações essenciais à orientação de intervenções educativas e assistenciais no âmbito das Casas de Acolhimento. Diante disso, o presente estudo tem como finalidade: caracterizar, quanto aos aspectos biopsicossociais, a população de crianças e adolescentes em situação de acolhimento institucional em um município da região centro-oeste de Minas Gerais.

\section{MÉTODOS}

Trata-se de um estudo do tipo exploratório, descritivo que teve como cenário de investigação duas Casas de Acolhimento Infanto-juvenil localizadas em um munícipio do Estado de Minas Gerais e como participantes crianças e adolescentes institucionalizados. Este estudo é proveniente de um pesquisa ampliada em execução desde 2017, intitulada "Acolhimento institucional e extensão universitária: contribuições para a promoção da saúde de crianças e adolescentes institucionalizados e para a formação profissional dos discentes" que tem interface com Programas de Iniciação Científica e com o Programa de extensão ACOLHER desenvolvido por estudantes, pesquisadoras e Residência Multiprofissional em Saúde do Adolescente de uma Universidade Pública Federal, localizada em Minas Gerais.

A coleta de dados ocorreu por meio de um roteiro semiestruturado construído à luz do questionário da Pesquisa Nacional de Saúde do Escolar (PeNse), versão 2015. A escolha do PeNse como instrumento de referência para a elaboração do roteiro de coleta deve-se à sua abrangência de questões sobre aspectos socioeconômicos; contexto familiar; hábitos alimentares; prática de atividade física; experimentação e consumo de cigarro, álcool e outras drogas, entre outros aspectos.

As questões supracitadas foram consideradas no instrumento elaborado para esse estudo com destaque para as seguintes variáveis: (I) Sociodemográficas: idade, sexo, cor ou raça, escolaridade dos pais, tipo de trabalho e acesso à internet; (II) Relacionadas ao uso de cigarro: experimentação de cigarro pelo menos uma vez, idade do primeiro contato, número de dias que usou o cigarro nos últimos 30 dias, tentativa de parar de fumar nos últimos 12 meses, qual de seus pais ou responsáveis fuma e qual seria a reação da sua família se ela soubesse que você fuma; (III) outras variáveis relacionadas a alimentação, atividade física, drogas ilícitas.

O roteiro elaborado buscou atender as particularidades que envolvem a faixa etária definida para o estudo composta de crianças a partir de sete anos e adolescentes até 17 anos incompletos. Participaram do estudo 11 crianças com idades entre sete e 11 anos e 12 adolescentes entre 12 e 17 anos.

Houve perda de quatro participantes, por motivos de adoção, volta para a família e trabalho. Foram excluídas crianças menores de sete anos de idade devido a possíveis dificuldades para interpretação dos questionamentos feitos.

A coleta ocorreu no período de outubro a dezembro de 2018 , em horários previamente agendados com as coordenadoras das Casas de Acolhimento e com as crianças e adolescentes participantes.

Para a análise dos dados, com o auxílio do Excel, foram estimadas frequências simples das variáveis idade, sexo, raça/cor, escolaridade materna. Para a escolaridade materna foram usadas as categorias: nenhuma ou fundamental incompleto, fundamental completo ou ensino médio incompleto; ensino médio completo ou superior incompleto, e superior completo. As variáveis que incluíram alimentação saudável e não saudável, atividade física, uso de cigarro, álcool e drogas, segundo sexo, também foram analisadas. 
Foram respeitados os preceitos éticos da pesquisa em todas as suas etapas. O estudo foi direcionado ao Comitê de Ética em Pesquisa com o Certificado de Apresentação para Apreciação Ética (CAAE) de $n^{\circ} 73975417.1 .0000 .5545$ e parecer $n^{\circ} 02.271 .545$ aprovado em 12/09/2017. Todos os participantes foram previamente informados acerca da pesquisa, de suas etapas, riscos e benefícios e em seguida foram assinados o Termo de Assentimento Livre e Esclarecido (TALE), no caso das crianças e adolescentes e o Termo Consentimento Livre e Esclarecido (TCLE), sendo o segundo assinado pelas coordenadoras das Casas de Acolhimento.

\section{RESULTADOS E DISCUSSÃO}

A distribuição das crianças e adolescentes institucionalizados participantes do estudo quanto à idade revela que no conjunto, todas as participantes do sexo feminino (100\%) da instituição têm idade menor ou igual a 13 anos, sendo $50 \%$ com 11 anos e $50 \%$ com nove anos. Participantes do sexo masculino mais da metade $52 \%$ tem idade menor ou igual que 13 anos, seguida de $24 \%$ com 14 anos de idade. Quanto a característica cor da pele, observa-se a ocorrência de maiores proporções de pardos $(81 \%)$ e brancos $(19,0 \%)$ para o conjunto dos participantes do sexo masculino e para sexo feminino a distribuição foi de $50 \%$ para parda e branca (Tabela 1).

Tabela 1 - Distribuição dos participantes segundo idade, raça/cor, sexo no conjunto das casas de acolhimento localizadas em município de Minas Gerais, no período de outubro a dezembro de 2018.

\begin{tabular}{lcccc}
\hline \multicolumn{1}{c}{ Variáveis } & Feminino (\%) & Número & Masculino (\%) & Número \\
\hline Idade em anos & & & & \\
\hline$<$ ou $=13$ & 100 & 2 & 52 & 11 \\
13 & - & - & 5 & 1 \\
14 & - & - & 5 & 5 \\
15 & - & - & 14 & 1 \\
16 e mais & - & - & 81 & 3 \\
\hline Cor ou raça & 50 & 1 & 19 & 17 \\
Parda & 50 & 1 & & 4 \\
Branca & & &
\end{tabular}

Fonte: Costa CC, et al., 2018.

A variável cor/raça retratada neste estudo corrobora com os dados do Instituto Brasileiro de Geografia e Estatística (IBGE) visto que a pesquisa realizada pelo instituto mostra que, entre 2012 e 2016, a participação percentual dos brancos na população do país caiu de $46,6 \%$ para $44,2 \%$, enquanto a participação dos pardos aumentou de $45,3 \%$ para $46,7 \%$ e a dos pretos, de $7,4 \%$ para $8,2 \%$ (IBGE, 2016).

Quanto à escolaridade materna, $67 \%$ dos participantes do sexo masculino e $100 \%$ do sexo feminino não sabem informar. Daqueles que sabem informar a escolaridade materna, observa-se que $9 \%$ das mães apresentam nenhum ensino ou fundamental incompleto, $19 \%$ possuem ensino fundamental completo ou médio incompleto e nenhuma mãe apresenta ensino superior completo (Tabela 2).

Tabela 2 - Distribuição dos participantes segundo sexo e escolaridade materna no conjunto das casas de acolhimento localizadas em município de Minas Gerais, no período de outubro a dezembro de 2018.

\begin{tabular}{lcccc}
\hline \multicolumn{1}{c}{ Variáveis } & Feminino (\%) & Número & Masculino (\%) & Número \\
\hline Escolaridade materna & & & & \\
\hline Não sabe informar & 100 & 2 & 67 & 14 \\
Nenhum ou Fundamental incompleto & - & - & 9 & 2 \\
Fundamental completo ou médio incompleto & - & - & 19 & 4 \\
Médio completo ou superior incompleto & - & & 5 & 1 \\
Superior completo & - & & - & - \\
\hline
\end{tabular}

Fonte: Costa CC, et al., 2018. 
A condição de vulnerabilidade socioeconômica familiar associada à baixa escolaridade dos genitores, separação conjugal como também a ocorrência de morte e pobreza é considerada na literatura como fator de risco para a saúde mental além de contribuir para comportamentos de risco e diminuição do desempenho escolar. Esse ambiente físico e social prejudicado favorece a manutenção das desigualdades sociais em saúde, principalmente quando são vivenciados na adolescência (SCHREIER HMC e CHEN E, 2013)

Neste estudo, também foram considerados a ocorrência dos indicadores de risco e proteção à saúde como variáveis para a análise do perfil biopsicossocial das crianças e adolescentes (Tabela 3).

Tabela 3 - Frequência (\%) de fatores de risco e proteção à saúde apresentados pelos participantes segundo sexo no conjunto das casas de acolhimento localizadas em município de Minas Gerais, no período de outubro a dezembro de 2018.

\begin{tabular}{lcccc}
\hline \multicolumn{1}{c}{ Variáveis } & Feminino (\%) & Número & Masculino (\%) & Número \\
\hline Consumo alimentar & & & & \\
\hline Feijão & 100 & 2 & 100 & 21 \\
Fruta & 100 & 2 & 100 & 21 \\
Guloseima & 50 & 1 & 81 & 17 \\
Refrigerante & 50 & 1 & 86 & 18 \\
\hline Atividade Física & & & 53 & 11 \\
\hline Ativo & 0 & - & 10 & 2 \\
Hábito sedentários em frente à TV & 50 & 1 & & \\
\hline Hábito de fumar & & & 5 & 1 \\
\hline Experimentação de cigarro & 0 & - & 53 & 11 \\
Fumante atual & 0 & - & 5 & 1 \\
\hline Consumo de álcool e drogas & & - & 15 & 3 \\
\hline Experimentação de bebida alcóolica & 0 & - & & \\
Consumo atual de bebida alcóolica & 0 & & & \\
Uso de drogas ilícitas & 0 & &
\end{tabular}

Fonte: Costa CC, et al., 2018.

Com relação aos alimentos marcadores de alimentação saudável, todas as crianças e adolescentes informaram consumir feijão em pelo menos cinco dias da semana (100\%). O consumo regular de frutas frescas foi alto (100\%), semelhante entre meninos e meninas. Na pesquisa nacional de saúde do escolar (PeNSE), ano base 2015, constatou-se que o consumo de frutas frescas e de feijão, entre os estudantes brasileiros do $9^{\circ}$ ano foi de $32,7 \%$ e $60,7 \%$, respectivamente.

A divergência entre os resultados do estudo e aqueles apresentados pela PENSE (2015) justifica-se pelo fato de que as crianças e adolescentes ao serem acolhidas devem ter garantidos todos os seus direitos, inclusive de alimentação e hábitos de vida saudáveis. (BRASIL, 2009).

As casas de acolhimentos, consideradas como locais que devem zelar pela proteção e manutenção da saúde das crianças e adolescentes institucionalizados, ao preparar as refeições buscam atender às necessidades nutricionais das crianças e adolescentes, além de serem instituídas práticas de alimentação saudável com a valorização do consumo diário de frutas, hortaliças, dentre outros alimentos. Hábitos alimentares saudáveis na infância e adolescência são considerados como potencializadores de manutenção na vida adulta e idosa (MALTA et al, 2010; IBGE, 2016), bem como permitem inferir que o consumo regular de frutas e hortaliças favorecem o controle do peso, além de funcionar como fatores de proteção para doenças cardiovasculares e diabetes tipo 2.

Entretanto, em relação as bebidas, os refrigerantes foram os mais consumidos (86\%), especialmente pelos meninos, fato também observado em relação às guloseimas que aparecem em destaque (81\%) no sexo masculino. 
O hábito de fumar e consumo de álcool e drogas foi ausente nas crianças e adolescentes do sexo feminino. Já no sexo masculino houve $43 \%$ de experimentação de cigarro, $53 \%$ de experimentação de bebida alcoólica e 15\% de uso de drogas ilícitas, com menção ao uso de maconha. Crianças e adolescentes institucionalizados tem suas histórias de vida experiências que as tornam expostas a diversas situações de risco dentre as quais está o uso de drogas. Em estudo realizado por Furlan e Souza (2014) a dependência química dos pais e envolvimento com as drogas ocupou a primeira causa de institucionalização de crianças e adolescentes em uma casa de acolhimento de munícipio de Piracicaba. Quando em situação de acolhimento, estudos tem demonstrado que a procura das drogas representa o principal motivador da evasão dos adolescentes das casas de acolhimento (ACIOLI RML, et al. 2018; LEMOS e MENESES, 2002).

Políticas nacionais relacionadas à promoção da saúde de adolescentes, propostas pelo Ministério da Saúde, estão em processo de estruturação no âmbito dos governos federal, estadual e municipal. Essas políticas têm como finalidade promover, por meio de metodologias problematizadoras, ações que favoreçam o autocuidado e o empoderamento dos adolescentes; fortaleçam sua autoestima e desenvolvem perspectivas de futuro favoráveis (BRASIL EGM et al, 2017)

\section{CONCLUSÃO}

A infância e a adolescência emergem como grupos que apresentam singularidades que os definem e diferenciam dos demais grupos assistidos no Sistema Único de Saúde. Este estudo revela que o contexto familiar composto de situações de risco e vulnerabilidades permite que as crianças e adolescentes tenham suas histórias de vida permeadas por condições socioeconômicas precárias, bem como aproximações diretas e indiretas com consumo de drogas lícitas e ilícitas.

\section{FINANCIAMENTO}

Agradecimento a Fundação de Amparo à Pesquisa do Estado de Minas Gerais (FAPEMIG) responsável pelo financiamento da iniciação científica do projeto de pesquisa intitulado: "Construindo o perfil biopsicossocial de crianças e adolescentes institucionalizados: aprendendo a olhar nas entrelinhas" aprovado no Edital 009/2017/PROPE - PIBIC/FAPEMIG/UFSJ.

\section{REFERÊNCIAS}

1.ACIOLI RML, et al. Avaliação dos serviços de acolhimento institucional de crianças e adolescentes no Recife. Ciência \& Saúde Coletiva, 2018; 23(2): 529-542.

2. BRASIL. Estatuto da Criança e do Adolescente, Câmera dos Deputados, Lei no 8.069, de 13 de julho de 1990. DOU de 16/07/1990 - ECA. Brasília, DF. p. 36.

3. BRASIL. Conselho Nacional dos direitos da criança e do adolescente. Orientações técnicas: Serviços de acolhimento para crianças e adolescentes. Ministério do Desenvolvimento Social e Combate à fome. Brasíliia, DF, 2009. p. 169.

4. BRASIL EGM, et al. Adolescent health promotion and the School Health Program: complexity in the articulation of health and education. Rev Esc Enferm USP. 2017

5. FERREIRA VVF, LITTIG PMCB, VESCOVI RGL. Crianças e adolescentes abrigados: perspectiva de futuro após situação de rua. Psicologia e sociedade, 2014; 26:165-174.

6.FUKUDA CC, PENSO MA, SANTOS BR. Configurações sociofamiliares de crianças com múltiplos acolhimentos institucionais. Arq. bras. psicol., Rio de Janeiro, 2013; 1:70-87.

7. FURLAN V, SOUSA TRP. Família, Acolhimento Institucional e Políticas Públicas: um estudo de caso. Psicologia Política. 2014; 14(31): 499-516.

8. IBGE. Instituto Brasileiro de Geografia e Estatística. Pesquisa nacional de saúde do escolar: 2015. Rio de Janeiro: IBGE, 2016. $132 \mathrm{p}$.

9. LEMOS MS, MENESES HI. A Avaliação da Competência Social: Versão Portuguesa da Forma para Professores do SSRS. Psicologia: Teoria e Pesquisa, 2002; 18(3): 267-274.

10. MALTA DC, et al. Prevalência de fatores de risco e proteção de doenças crônicas não transmissíveis em adolescentes: resultados da Pesquisa Nacional de Saúde do Escolar (PeNSE), Brasil, 2009. Ciênc. saúde coletiva, Rio de Janeiro, 2010; 15(2): 3009-3019. 
11. MALTA DC, et al. Tendência dos fatores de risco e proteção de doenças crônicas não transmissíveis em adolescentes, Pesquisa Nacional de Saúde do Escolar (PeNSE 2009 e 2012). Rev. bras. epidemiol. 2014; 17(1): 77-91.

12. MALTA DC, et al. Política Nacional de Promoção da Saúde (PNPS): capítulos de uma caminhada ainda em construção. Cienc Saude Coletiva, 2016; 21(6): 83-94.

13. OlIVEIRA MM, et al. Características da Pesquisa Nacional de Saúde do Escolar - PeNSE. Epidemiol. Serv. Saúde. 2017; 26(3): 605-616.

14. SCHREIER HMC, CHEN E. Socioeconomic status and the health of youth: a multi-level, multi-domain approach to conceptualizing pathways. Psychological Bulletin, Washington, DC: American Psychological Association, 2013; 139(3): 606-654.

15. WHO. World Health Organization. Inequalities young people's health: key findings from the Health Behaviour in Schoolaged Children (HBSC) 2005/2006 survey fact sheet. Copenhagen: World Health Organization; 2008.

16. ZAPPE JG, DELL'AGLIO DD. Adolescência em diferentes contextos de desenvolvimento: risco e proteção em uma perspectiva longitudinal. Psico (Porto Alegre), Porto Alegre. 2016; 47(2): 99-110. 